\title{
Energy Efficient Low Latency Multi-issue Cores for Intelligent Always-On loT Applications
}

\author{
Joonas Multanen $^{1}$ (D) $\cdot$ Heikki Kultala ${ }^{1} \cdot$ Kati Tervo $^{1} \cdot$ Pekka Jääskeläinen ${ }^{1}$ \\ Received: 2 May 2019 / Revised: 29 June 2020 / Accepted: 6 July 2020 / Published online: 26 July 2020 \\ (C) The Author(s) 2020
}

\begin{abstract}
Advanced Internet-of-Things applications require control-oriented codes to be executed with low latency for fast responsivity while their advanced signal processing and decision making tasks require computational capabilities. For this context, we propose three multi-issue core designs featuring an exposed datapath architecture with high performance, while retaining energy-efficiency. These features are achieved with exploitation of instruction-level parallelism, fast branching and the use of an instruction register file. With benchmarks in control-flow and signal processing application domains we measured in the best case $64 \%$ reduced energy consumption compared to a state-of-the-art RISC core, while consuming less silicon area. A high-performance design point reaches nearly $2.6 \mathrm{GHz}$ operating frequency in the best case, over $2 \times$ improvement, while simultaneously achieving a $14 \%$ improvement in system energy-delay product.
\end{abstract}

Keywords Low power · Instruction stream · Energy-efficiency · Instruction register file · IoT · Always-on · RISC-V · TTA $\cdot$ Exposed datapath $\cdot$ Transport Triggered Architecture

\section{Introduction}

It is estimated, that the information and communication technology (ICT) sector will consume up to $20 \%$ of global energy production by 2025 [1]. From an environmental point of view, there are estimates that around $14 \%$ of total greenhouse gas emissions emerge from the ICT sector by 2040 [2]. The era of Internet-of-Things (IoT) and its increasing demands on computational complexity are expected to result in the introduction of billions of compute devices. Many of these small form factor devices

Joonas Multanen

joonas.multanen@tuni.fi

Heikki Kultala

heikki.kultala@tuni.fi

Kati Tervo

kati.tervo@tuni.fi

Pekka Jääskeläinen

pekka.jaaskelainen@tuni.fi

1 Faculty of Information Technology and Communication Sciences, Tampere University, Tampere, Finland are battery-powered or use energy harvesting for their power supply, requiring energy-efficient and low power operation.

While maintaining low energy consumption, devices such as always-on surveillance cameras, small drones, and sensor nodes, are required to react to events and perform demanding signal processing and artificial intelligence tasks, and also to handle external events with low control code execution latency. Besides their low power and energy consumption requirements, this calls for the devices to be highly performance scalable.

For maximal energy-efficiency, fixed function accelerators are typically used. Compared to programmable devices, their hardware is optimized at design-time to match predefined requirements. This allows removing instruction delivery overheads and tailoring the datapath, resulting in high computational capability and energy efficiency in small chip area. The clear drawback is that the accelerators perform poorly or not at all with tasks not defined at design time. Moreover, their design, optimization and verification is a costly, time consuming process requiring manual effort [3, 4]. In contrast, software programmable devices offer flexibility [5] in terms of non-predefined tasks and reduce the design cost and time with reusable compute and logic elements, but incur overheads due to the flexible software based control. 
In this article, we propose energy-efficient, programmable processor cores for always-on applications. The cores feature fast branching and efficient exploitation of exposed datapath instruction-level parallelism (ILP) to achieve high performance. In order to reduce the energy overhead of instruction delivery, a compiler-controlled instruction register file (IRF) is used. The cores are compared to a publicly available LatticeMico32 (LM32) [6] core offered by Lattice Semiconductor and zero-riscy [7], a RISC-V [8] ISA implementation using benchmarks representing both control oriented and signal processing tasks. All the cores are evaluated on a $28 \mathrm{~nm}$ ASIC standard cell technology.

For this article we extended our previous conference paper [9] with the following additions:

- The previously proposed LoTTA design was adapted to two additional design targets: high energy-efficiency and high clock frequency.

- More accurate results obtained by place \& route as opposed to original synthesis results.

- A comparison to a state-of-the-art low power RISC-V based core, zero-riscy was added.

- Extended textual presentation with detail added specifically to parts that were pointed out by the conference paper reviews.

The article is organized as follows. Section 2 overviews related work. Section 3 describes the three core variants along with an explanation of their underlying architecture and programming model. Section 4 details the concepts of instruction register files and the two implementation variations used in this work. Section 5 presents the evaluation results, and Section 6 concludes the article.

\section{Related Work}

The emerging era of IoT has resulted in plenty of processor proposals suitable for always-on energy constrained scenarios. The approaches vary from generic to highly domainspecific. SleepWalker [10] and the IoT SoC [11] proposed by Klinefelter et al. are targeted for scenarios, where energy delivery is difficult and solutions such as energy-harvesting are required to power the device, such as wireless sensor networks. The latter is targeted for biomedical applications and includes domain-specific accelerators to maximize energy-efficiency with the trade-off of computational flexibility. Recryptor [12] is an ARM Cortex-M0 based IoTtargeted cryptoprocessor, where energy-efficiency stems from near-threshold voltage operation and in-memory computing. Senni et al. [13] leverage the non-volatility and low leakage power consumption of magnetic random access memory (MRAM) to reduce processor energy consumption when idling. Wang et al. [14] propose a dual-core processor system, where one core has high performance and the second core has relatively low performance, but is $3 \times$ more energy-efficient. The authors utilize their two-core system with a proposed energy-aware task scheduler. Roy et al. [15] utilize sub-threshold voltage operation in their implementation of MSP430 targeted for IoT domain and especially for biomedical applications.

The recently proposed zero-riscy [7] implements the popular RISC-V instruction set architecture (ISA). It includes lightweight instruction prefetching and compressed instructions, and thus seems to be closest to our work in terms of processing capabilities and target context. Since its implementation is available as open source, it was picked for closer comparison to this article.

Another openly available alternative is the LatticeMico32 [6]. It is a 32-bit microprocessor core with a Harvard architecture and a RISC ISA, with optional configurable multiplier and shifter units.

Sleepwalker [10] is also close to our work. It uses adaptive voltage scaling along with sub-threshold voltage operation to achieve energy-efficiency. Both of these techniques could be applied on top of our proposed cores to improve the energy efficiency even further. In fact, there is already previous work of a chip implementation of an application-specific sub-threshold voltage transporttriggered architecture (TTA) core developed using the same tools and the processor template as the cores proposed in this paper [16].

The most apparent feature differentiating the proposed processor is its transport-triggered programming model, where data forwarding is controlled by software, eliminating the forwarding logic hardware overheads and supporting instruction-level parallelism with simpler register files. Maxim Integrated commercialized a TTA-based microcontroller in 2004. The microprosessor called MAXQ [17] uses the transport programming model to simplify the processor structure and is optimized for control oriented codes characterized by heavy branching. From their white papers we observe that the key difference of MAXQ to the cores presented in this article are is its $16 \mathrm{~b}$ scalar data path, while the proposed cores include a $32 \mathrm{~b}$ data path and integrate additional features to reduce the instruction stream energy footprint and to support higher maximum clock frequencies combined with instruction-level parallelism.

\section{Proposed Cores}

In previous work [9], we designed the Low-power Transport Triggered Architecture (LoTTA) by using the TTA-based Co-Design Environment (TCE) [18] processor design tools. Figure 1 shows LoTTA in the TCE processor designer with 


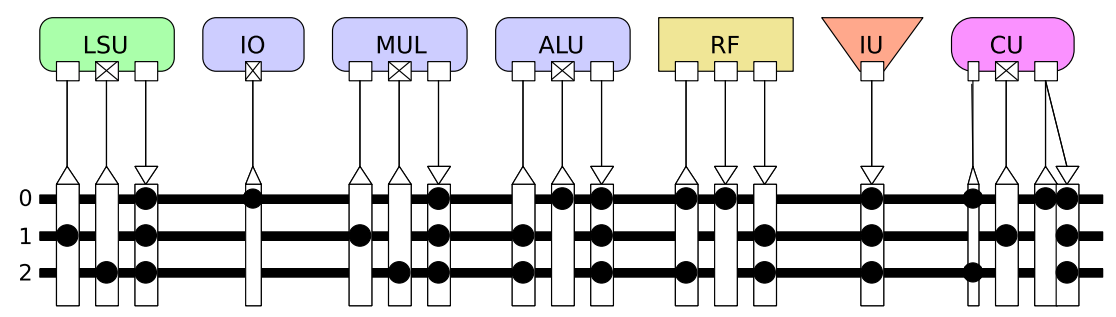

Figure 1 TCE view of the function unit and interconnection architecture common to the proposed cores. LSU—load-store unit, IO-standard input-output, MUL—multiplier, ALU—arithmetic logic unit, RF—register file, IU—immediate unit, CU—control unit.

an overview of the databus interconnection network and individual function units. The same interconnection network and function unit organization was used as a basis for all three of the proposed cores. As increasing the number of connections in the interconnection network increases the logic required, often ends up in the critical path, and widens the instruction width of the bus transport programmed architecture, it is important to prune the interconnection network carefully to a minimal level, that can still maintain good performance.

The core intends to combine qualities needed for fast execution of control code along with signal processing, and is designed with the mindset to support various workloads. Therefore, special purpose function units were not utilized in addition to generic load-store units, register files, ALUs and multipliers. This mindset was continued in the other two proposed cores. Operations implemented in the cores are listed in Table 1. The cores utilize software based data forwarding enabled by a transport-triggered programming model which result in a very simple and energy efficient control unit and reduced register file microarchitecture complexity.

This paper extends our previous work by proposing two design points modified from the original processor core. For the purposes of this paper we set the instruction bit width

Table 1 Operations implemented in the proposed cores.

\begin{tabular}{ll}
\hline Function unit & Operations \\
\hline LSU & $\{8,16 \& 32\}$ bit load/store \\
IO & write to stdout \\
MUL & multiply \\
ALU & abs, add, and, eq, gt, gtu, ior, \\
& max, maxu, min, minu, ne, neg, shl, \\
& shr, shru, sub, sxhw, sxqw, xor \\
RF & read/write register \\
IM & load/store 32 bit immediate \\
CU & jump, call, $\{\text { bz, bnz }\}^{\text {a }}$ \\
\hline
\end{tabular}

${ }^{\mathrm{a}}$ LoTTA core uses predicated execution instead of conditional branch operations to the exact number derived from the core features, even if it is not a power of two or even byte-aligned. Instead of extending the instruction width to a byte alignment or a power of two, we assume that in an application-specific processor, the memory architecture can be customized for maximal energy-efficiency. If needed, the instruction width can be aligned depending on the use case.

Next we describe the underlying architecture and programming model in the proposed cores, followed by details of the core variants.

\subsection{Transport Triggered Architecture}

Transport triggered architecture (TTA) belongs to the family of "exposed datapath architectures", where the datapath interconnection network is controlled by the programmer. TTAs feature a long instruction word to describe instruction-level parallelism in applications. Compared to similar very long instruction word (VLIW) processors, having an exposed datapath allows TTAs to transport data values between function units via software bypassing. This has the advantage of not requiring hardware to detect data hazards when forwarding. Optionally registered input and output ports of function units reduce unnecessary register file traffic, allowing TTAs to reach equal performance with a simpler register file, with fewer physical ports when compared to a VLIW [19].

An understudied advantage of TTA is its simplified execution pipeline. Similar to traditional RISC processors with multiple pipeline stages, the first stages in TTA pipeline are instruction fetch and decode. However, whereas different types of operations are fixed to a distinct stage in a RISC pipeline, TTAs can perform any type of operation after the decode stage. These include memory operations, register file transfers, or any kind of ALU or custom operations. This is illustrated in Fig. 2, where the three different TTA execution pipelines of the proposed cores are compared against a classic RISC pipeline and two of its variants implemented in the reference cores.

While TTAs have been targeted and studied extensively as overcoming some of the shortcomings of VLIW processors since the 1990s, they were first originally introduced 


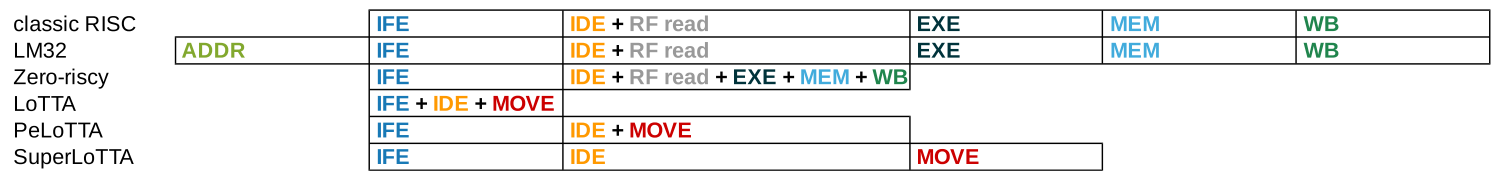

Figure 2 Execution pipelines stages of the evaluated cores compared with a classic RISC pipeline organization.

as control processors in the 1970s [20]. Here, only a single data move instruction was used to transport data between memory mapped control registers.

The pipeline flexibility, simplified register files and elimination of data forwarding hardware allow TTAs to merge the instruction fetch and decode stages, while retaining a high clock frequency. This results in fast branching and, therefore, efficient execution of control code. This is done while still providing ILP required by more complex algorithms.

The next sections describe the details of the proposed cores. Execution pipelines of the cores are compared in Fig. 2. Comparison of the differences in the architecture of the proposed cores is listed in Table 2.

\subsection{LOTTA}

In the LoTTA core, the primary design goal was to minimize the use of hardware resources and to minimize branching delays for fast execution of control-oriented code [9], while maintaining good signal processing performance. For fast branching, we merged the instruction fetch, decode and move stages into the same pipeline stage. This is feasible thanks to the straightforward, yet efficient fetch and decode logic of the TTA programming model. LoTTA utilizes predicated execution for streamlining conditional codes. However, together with the merged fetch and decode, this results in a long critical path delay [9] as the instruction SRAM implemented in the processor system ends up in the critical path.

\subsection{PeLoTTA}

PeLoTTA is a clock frequency optimized high-performance version of LoTTA. Here, the target was to optimize the maximum clock frequency, while still supporting the fast branches and energy-efficient operation of the original
LoTTA. To achieve this, the instruction pipeline was split from one to two stages; the first, instruction fetch stage, retrieves an instruction from the memory, with decode and the move/execute in the second.

In order to further optimize the clock frequency, the predicated execution of LoTTA, which ended up in the critical path [21], was replaced with conditional branch operations branch equal to zero (bz) and branch not equal to zero (bnz). This also reduced the instruction width, as the boolean RF used to hold predicate values was removed, saving bits in the instruction word that were used to control it. Likewise, the instruction decode unit was simplified, as the predicate evaluation was removed.

\subsection{SuperLoTTA}

As a design point targeting maximum operating clock frequency even with the trade-off in additional operation execution latencies that need to be dealt with in the software side, we designed the SuperLoTTA core. In order to achieve this design target, we added extra pipeline registers to the ALU, load-store unit and multiplier unit, isolating them from the interconnection network. The same was done to the control unit, which allowed to isolate instruction memory from the critical path.

As with PeLoTTA, to further optimize the clock frequency we added conditional branch operations. This allowed us to simplify the decoder logic compared to full predicated execution capabilities.

\section{Instruction Register File}

While "exposed datapath processors" have benefits in terms of simplified hardware, they incur a trade-off in increased compiler complexity due to the additional programmer responsibilities. Moreover, increased programmer control
Table 2 Feature comparison of the proposed cores.

\begin{tabular}{llllllll}
\hline & $\begin{array}{l}\text { Instr. } \\
\text { width }\end{array}$ & $\begin{array}{l}\text { Branch } \\
\text { delay slots }\end{array}$ & $\begin{array}{l}\text { Predicated } \\
\text { execution }\end{array}$ & $\begin{array}{l}\text { Cond. branch } \\
\text { operations }\end{array}$ & $\begin{array}{l}\text { ALU } \\
\text { delay }\end{array}$ & $\begin{array}{l}\text { Optional } \\
\text { IRF style }\end{array}$ & $\begin{array}{l}\text { Design } \\
\text { target }\end{array}$ \\
\hline midrule LoTTA & 49 & 0 & $\checkmark$ & & 1 & basic & Branch delay \\
PeLoTTA & 45 & 1 & & $\checkmark$ & 1 & improved & Energy-efficiency \\
SuperLoTTA & 43 & 3 & & $\checkmark$ & 2 & & Clock frequency \\
\hline
\end{tabular}


translates to a wider instruction word and increased code size in general, which conflicts with the need to optimize the instruction stream's energy consumption. [22]

Instruction memory hierarchies typically employ small storages close to the core to store temporally and spatially related code, while larger but slower storages are kept farther away. Traditionally, caches controlled by hardware have been used as lower level instruction storages. Their operation requires keeping track of values present in the cache with separate tag bits, which need to be checked upon a cache read. If the value requested is not present in the cache, execution stalls while the cache miss is resolved from higher levels in memory hierarchy.

Caches can be integrated into a system with relatively small effort, due to being hardware-controlled, but with the cost of control logic overhead. This overhead can be removed, if control is moved to software, such as in an instruction register file. An IRF is explicitly instructed to fetch new instructions, with the additional benefit of possibility to separate the fetching of instructions from their execution. While this allows a type of speculative fetching, the compiler is required to efficiently decide the placement of special IRF load instructions for good performance. With their fine-grained control qualities, IRFs have been shown to be suitable for low power architectures. [23]

IRFs require certain design choices for their implementation. Bypassing allows instructions to be read directly from the next level in memory hierarchy without first storing them into the IRF. However, the access time of the larger instruction memory unit is likely to add to the design's critical path. With a smaller unit such as IRF, the effect on the critical path is smaller. This requires analyzing the application for instructions, that are beneficial to be executed from the IRF such as loops, and instructions whose writing to the IRF would incur an energy overhead in relation to their execution amount. In the latter case, bypassing the instruction would be preferred.

Common to both of the two IRF design variations we used in our cores introduced in the next section, IRF execution starts with a compiler-inserted header instruction, which contains the number of entries to fill into the IRF. At compile time, the instruction scheduler groups sequential instructions into IRF instruction windows. These are groups, that can simultaneously exist in the IRF, and are indicated by the header instructions.

\subsection{Basic IRF}

The basic IRF from our original work [9] is described in Fig. 3. Two program counter registers are utilized here: one for addressing the global instruction memory address space and another for addressing IRF entries. In cases where execution does not fall through from the IRF and a conditional branch exits IRF execution, instructions in the branch not taken would not be executed. To eliminate unnecessary fetching, the IRF is accessed using presence bits, which use a single bit per IRF entry to indicate, whether an instruction has been fetched or not. If the presence bit is not set, the instruction is fetched and then executed. The presence bits are reset at the start of a new IRF window execution. This differs from the state-of-the-art work [23], where no presence bits are used.

The presence bits also prevent cases, where an instruction branches forward inside the IRF and the target instruction is not fetched. Without presence bits the compiler would be forced to discard every instruction window containing forward jumps from being placed to the IRF.

As a design choice, we implement IRF bypassing to avoid writing instruction with low execution counts to the IRF while simultaneously maintaining zero delay slot branching. In other words, we allow execution of code directly from the next level in the instruction memory hierarchy. To maintain good performance, this would require low access times for the next level in memory hierarchy. Otherwise, the maximum clock frequency would be limited by the memory access times. Previous work [23] avoids this issue by writing all instructions to the IRF and then executing them with no option for bypassing.

In order to not increase the instruction width due to the addition of new IRF control instructions, we implemented the special header instruction into the immediate control field of the TTA instruction, where enough free bit combinations were available. A comparator pre-decodes this field during the instruction fetch stage and stalls execution for one clock cycle, as the header instruction is only used by the instruction fetch unit and not executed by the rest of the core. At this time, length of the IRF instruction window and prefill amount are read from the instruction and stored into a register in the instruction fetch unit. Next instruction following the header is the first instruction in the instruction window.

To differentiate between global and IRF address spaces, we implement a new branch instruction targeting the IRF address space and use existing branch instructions to target the global address space. The unconditional irfjump instruction is only allowed when executing from the IRF and branches to a target inside the current instruction window. Regular branches are allowed anywhere in the code. Encountering them during IRF execution transfers execution into bypassing the IRF.

When IRF execution reaches the last instruction in the current instruction window or the last physical IRF entry and execution does not branch back into the IRF, execution is again transferred into bypassing the IRF with a fallthrough to the next code block. We implement these with hardware comparators in the instruction fetch unit. 
Figure 3 Instruction fetch unit of the proposed cores when using the basic IRF.

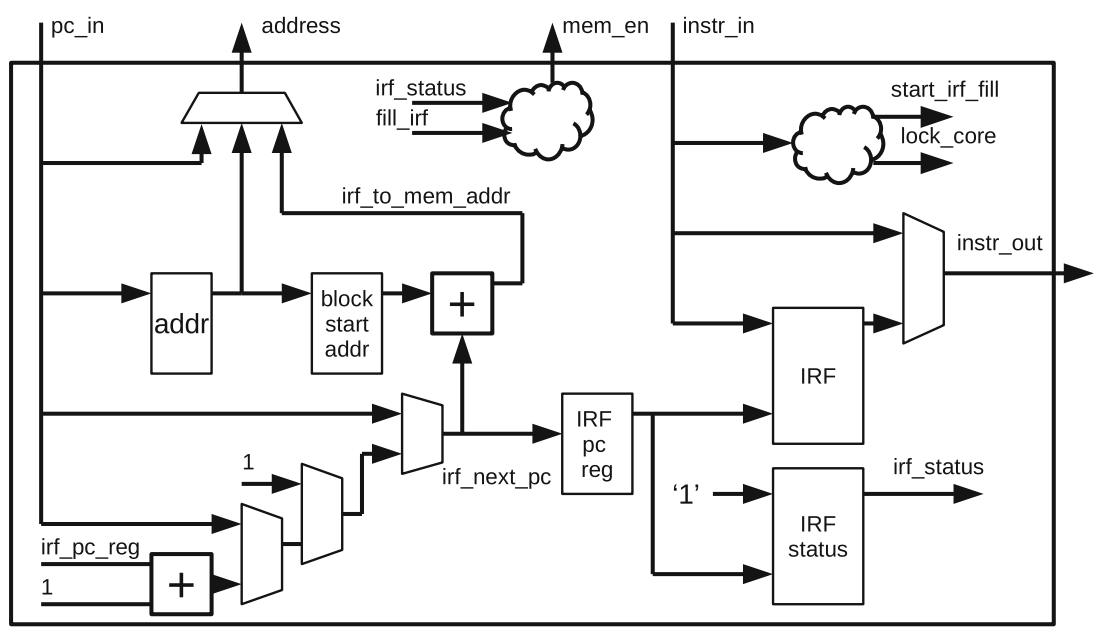

Adding the irfjump instruction to LoTTA core, where guarded execution is used for branching, increased the instruction size from 49 bits to 50 bits.

\subsection{Improved IRF}

According to our investigation [21] of IRF design choices, we optimize the IRF design in order to improve its energyefficiency and maximum operating clock frequency. The improved IRF is depicted in Fig. 4. We remove the hardware presence assurance, which guarantees that instructions are written into the IRF before trying to execute them and instead, we move the presence assurance to software. This removes the status bit register from the IRF.

During compilation, groups of instructions, instruction windows, to be placed into the IRF are analyzed for forward branches. Different from the basic IRF, if there are forward branches in the window, the compiler writes the number of instructions to prefill when starting IRF execution, into the header instruction, in addition to the current window size. The prefill guarantees the presence of instructions, removing the need to check each IRF entry individually.

As an optimization to improve clock frequency, we implement conditional branches into the IRF in addition to the irfjump operation: irfjumpnz and irfjumpz correspond to branch not equal to zero and branch equal to zero instructions. To minimize the hardware overhead, these are implemented as having 1-bit condition operand.

\subsection{Compiler Support}

Efficient utilization of the IRF requires consideration of two questions: which instructions should be executed from the IRF and when should the IRF be filled. Program control analysis in the context of IRFs has been previously studied in detail [24]. We implement compiler support primarily targeting loops and nested loops as these are typically the most heavily executed code structures in applications. Forming the instruction windows is done as a post-pass following the compiler instruction scheduling.
Figure 4 Instruction fetch unit of the proposed cores when using the improved IRF.

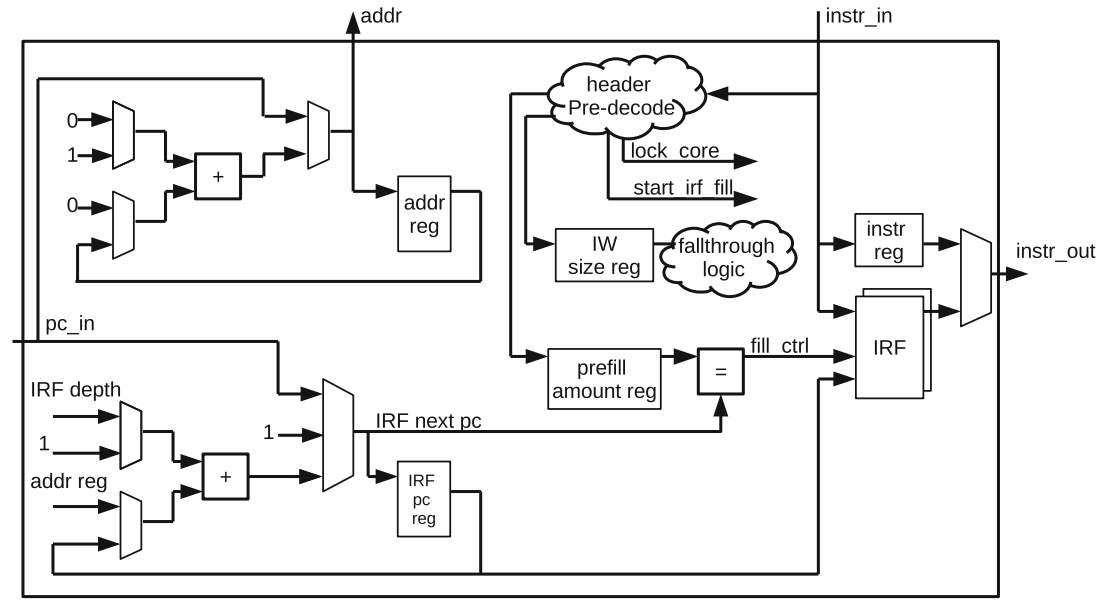


Our IRF instruction window allocation algorithm is presented in Fig. 5. Overall, on lines 3 to 9 the algorithm first splits program basic blocks (BBs) into blocks that fit into the IRF and merges them (lines 10 to 18 ) with two constraints:

1. Incoming jumps must target the first instruction of the instruction window.

2. Function calls are only allowed as the last instruction of an instruction window.

Respecting these constraints, upon encountering a function call, instruction windows are split at function calls, which are left as last instructions of a window. Returning from the function, execution continues from the next instruction address, which can contain a header instruction to start a new IRF instruction window, or start direct execution, bypassing the IRF.

Instruction windows with no backward branches are assigned to bypass the IRF on lines 19 to 26 . For instruction windows with backward branches, the window's first local

1: windowFitsToIRF: irfWindow.size + nextWindow.size < irfSize 2: canMergeIRFWindows: NOT irfWindow.endsInCall and
nextWindow has no incoming branches from outside these two windows and irfWindow.fitsToIR

3. for all basicBlocks in $C F G$ do

4: if basicBlock.size > irf Size then

5: $\quad$ split BB to irfSize

6: $\quad$ end if 1 create a new irfWindow for bb

8: $\quad$ place irfWindow into queue

9: end for

10: for all irfWindow in queue do

11: nextWindow $\leftarrow$ irfWindow.successor

12: if canMergeIRFWindows then

13: $\quad$ merge(irfWindow, nextWindow)

14: $\quad$ requeue(irf $W$ indow.predecessor)

15: else

rem

end if
end for

for all irfWindow in irfWindows do

if irf $W$ indow. hasNoBackwardBranches then

irf Window.set Bypassed

else

mark first local jump target

end if

end for

for all irfWindow in irfWindows do

if NOT irfWindow.isBypassed then

if NOT irfWindow.startsWithLocalBranchTarget then

split irfWindow from beginning

mark beginning as bypassed.

end if

f NOT irfWindow.hasLocalBranchAtEnd then

split irfWindow from end

mark the end as bypassed.

end if
end for

for all irfWindow in $\operatorname{irf} W$ indows do

if NOT irfWindow.isBypassed then

calculate stall cycles needed due to forward jumps

createHeaderInstruction (irf Window)

end if

end for

or all irfWindow in irfWindows do

if NOT irfWindow.isBypassed then

for all branch in irfWindow do

if branch.destination inside ir $f W$ indow then

convert branch to local branch

end if

end
end for

Figure 5 The IRF window allocation routine. branch target and the last local branch instruction are marked on lines 23 and 24, as these are effectively the loop boundaries. The algorithm first assigns inner loops into IRF instruction windows and continues to outer loops if the IRF capacity allows, as inner loops are expected to be program hot spots.

On lines 27 to 38 , code outside loop boundaries is removed from each instruction window, as this is code that is only expected to execute once each time an instruction window is programmed into the IRF.

When the instruction windows are completed, a header instruction is inserted into the beginning of the window on lines 39 to 44 . As we stall execution during IRF programming, the minimum amount of stall cycles required (prefill amount) is calculated according to forward branches in the instruction window. Instructions in IRF windows branching back into the window are converted into IRF branch instructions on lines 45 to 53 and their targets are converted into IRF indices. As a last step, all branch targets are fixed with respect to the additional header instructions.

As an improvement over our previous work [9], code that is executed only once is removed from the end of instruction windows. This further eliminates unnecessary writes to the IRF registers, saving energy. Moreover, support for conditional IRF branches is added. For the software presence assurance of instructions, on line 41 the minimum amount of stall cycles is calculated, if the IRF supports it. In this work, this is done for PeLoTTA core, which uses the improved IRF.

As the improved IRF is used in PeLoTTA core, IRF operations for the conditional branching were required. Irfjumpz and irfjumpnz, corresponding to the $b z$ and $b n z$ for regular branching were added to PeLoTTA, resulting in an increase of the instruction word from 43 to 45 bits.

\section{Evaluation}

The proposed cores and the reference cores were evaluated with benchmarks from two different use cases typical to always-on microcontrollers. All benchmarks were compiled with tcecc, the program compiler of TCE. Coremark [25] was used to evaluate the performance in control-oriented code. Competence in the other area of interest, digital signal processing (DSP), was evaluated with eight fixedpoint benchmarks from CHStone [26]. To verify the correct functionality of the $\mathrm{C}$ language benchmark programs, they were compiled for the processor and simulated using ttasim, TCE's instruction cycle-accurate simulator. Hardware level correctness was ensured by generating memory images from the compiled programs and then simulating them at register transfer level (RTL) with Mentor Graphics ModelSim 10.5. 
For the RISC cores, benchmarks were compiled with '-O3' optimization level. Zero-riscy uses a custom compiler of the PULPino project, based on GCC 5.4.0 and LatticeMico32 uses a custom compiler from Lattice Semiconductor, also based on GCC.

To compare the LoTTA core with a traditional multistage RISC architecture in our previous work, the LatticeMico32 core was used as a reference point. In addition to comparing LoTTA with LatticeMico32, in this extended work we compare PeLoTTA with a closer match, the recent lowpower zero-riscy [7] core. SuperLoTTA is also compared to zero-riscy and PeLoTTA, as its architecture is closer to the latter than that of LoTTA.

For comparison to LatticeMico32, hardware operations, their latencies, and register file sizes were matched in LoTTA. Arithmetic and logic operations in both cores were made very similar with separate multiplier and barrel shifter units. Register files in all cores are $32 \times 32$ bit, with two read ports and one write port. Physical area of the LatticeMico32 and LoTTA was validated to be roughly the same via ASIC place and route.

To evaluate our proposed core architecture with a recent low-power core intended for similar application domains, we used zero-riscy [7], a RISC-V core from the PULP [27] platform, and more specifically PULPino platform. For evaluation, we matched the number of pipeline stages, memory access delays and hardware operations closely in the PeLoTTA-ZR core, and adopted the latch-based register file used in zero-riscy. Of the two multiplier choices shipped with zero-riscy, a fast version was chosen over a slow variant. The PULPino framework was used to simulate the benchmarks on zero-riscy and generate switching activity files for power analysis after place and route. To provide a reference point of the PeLoTTA core also to LatticeMico32, we produced another subvariant with datapath components matching the those of LatticeMico32, named PeLoTTALM32 in the results.

Comparison of the architecture features of the proposed and reference cores is listed in Table 3.

In addition to LoTTA and PeLoTTA being evaluated individually, to evaluate the two IRF design alternatives,
LoTTA was also evaluated together with the basic IRF and PeLoTTA with the improved IRF.

All the cores evaluated were synthesized with Synopsys Design Compiler Q-2019.12. The process technology was $28 \mathrm{~nm}$ fully depleted silicon on insulator (FD-SOI) with $0.95 \mathrm{~V}$ voltage, with typical process corner and $25{ }^{\circ} \mathrm{C}$ temperature. After synthesis, place and route was performed with Synopsys IC Compiler II Q-2019.12. Switching activity interchange format (saif) files were produced with ModelSim 10.5 and used to estimate power for the synthesized designs.

\subsection{Code Size}

Compiled code sizes are listed in Table 4. As the zero-riscy core programs include operating system and debug related code, those were removed from the reported code sizes. Jpeg is not reported as the compiler could not fit it into the $32 \mathrm{kB}$ instruction memory set in the PULPino framework.

For the TTA cores with non-byte-aligned instruction widths, results are reported as total instruction bits as bytes. On average, LoTTA program size is $1.84 \times$ larger than the LatticeMico32 code. Coremark has the largest difference, where LoTTA instruction bit amount is $3.5 \times$ larger. When long instruction word processors cannot fill all of their instruction slots for each cycle, No-Operations (NOPs) are inserted into the code, bloating the code size. However, in aes, blowfish, mips and motion, LoTTA code size is smaller than that of LatticeMico32. This is due to loop unrolling and function inlining. These allow the wide TTA instructions to be efficiently utilized. Moreover, LatticeMico32 requires 32 bits for each instruction, whereas in LoTTA, a maximum of 3 instructions can fit into the 49 instruction bit wide instruction word per cycle, in the best case resulting in 16.3 bits on average per instruction slot. However, it is to be noted that the instructions in the two cores cannot be directly compared, as in the TTA programming model, individual data moves are controlled, whereas in the RISC model, the internal moves are controlled by hardware based on the input operations. In this sense, the TTA instructions more or less correspond to the internal data moves of a RISC processor.

Table 3 Architecture comparison of evaluated cores.

\begin{tabular}{|c|c|c|c|c|c|c|c|c|c|}
\hline & $\begin{array}{l}\text { Instruction } \\
\text { width (bits) }\end{array}$ & Architecture & $\begin{array}{l}\text { Issue } \\
\text { width }\end{array}$ & $\begin{array}{l}\mathrm{HW} \\
\mathrm{mul}\end{array}$ & $\begin{array}{l}\text { HW } \\
\text { div }\end{array}$ & $\begin{array}{l}\text { mul } \\
\text { latency }\end{array}$ & $\begin{array}{l}\text { div } \\
\text { latency }\end{array}$ & $\begin{array}{l}\text { Pipeline } \\
\text { stages }\end{array}$ & $\begin{array}{l}\text { Data mem. } \\
\text { access delay (cc) }\end{array}$ \\
\hline LoTTA & 49 & TTA & 3 & $\checkmark$ & & $3 \mathrm{cc}$ & & 1 & 1 \\
\hline PeLoTTA & 45 & TTA & 3 & $\checkmark$ & & $3 \mathrm{cc}$ & & 2 & 1 \\
\hline SuperLoTTA & 43 & TTA & 3 & $\checkmark$ & & $5 \mathrm{cc}$ & & 3 & 3 \\
\hline LatticeMico32 & 32 & RISC & 1 & $\checkmark$ & $\checkmark$ & $3 \mathrm{cc}$ & $34 \mathrm{cc}$ & 6 & \\
\hline zero-riscy & $32 \& 16$ & RV32IMC & 1 & $\checkmark$ & $\checkmark$ & $3 \mathrm{cc}$ & $36 / 0 \mathrm{cc}$ & 2 & 1 \\
\hline
\end{tabular}


Table 4 Cycle counts and program sizes.

\begin{tabular}{|c|c|c|c|c|c|c|c|c|c|}
\hline & coremark & adpcm & aes & blowfish & gsm & jpeg & mips & motion & sha \\
\hline & \multicolumn{9}{|c|}{ Cycle count } \\
\hline LatticeMico32 & 582818 & 88355 & 75184 & 798794 & 26798 & 3018543 & 27348 & 7757 & 677708 \\
\hline zero-riscy & 407036 & 149865 & 45268 & 942344 & 19872 & & 26099 & 3846 & 781108 \\
\hline LoTTA & 403416 & 81683 & 25690 & 673151 & 12745 & 8097755 & 23649 & 7797 & 543428 \\
\hline PeLoTTA & 521658 & 82814 & 34855 & 661369 & 13071 & 2361449 & 27735 & 9525 & 559836 \\
\hline \multirow[t]{2}{*}{ SuperLoTTA } & 802529 & 93691 & 41505 & 765160 & 16973 & 3275662 & 40023 & 7412 & 630984 \\
\hline & \multicolumn{9}{|c|}{ Code size (B) } \\
\hline LatticeMico32 & 12400 & 9396 & 17052 & 6820 & 6000 & 17520 & 3328 & 8620 & 4148 \\
\hline zero-riscy & 18292 & 9254 & 11660 & 5328 & 7290 & & 5724 & 4472 & 4712 \\
\hline LoTTA & 42979 & 16445 & 16372 & 6100 & 11851 & 50500 & 2597 & 6161 & 4269 \\
\hline PeLoTTA & 13787 & 12298 & 13406 & 5607 & 10283 & 42979 & 2887 & 9052 & 3704 \\
\hline SuperLoTTA & 18662 & 13949 & 16432 & 6440 & 14346 & 64775 & 3903 & 9858 & 4220 \\
\hline
\end{tabular}

Compared to LoTTA, benchmark code size for the PeLoTTA core is smaller in all but mips and motion benchmarks. This is mostly due to the smaller instruction word in PeLoTTA, 43 bits as opposed to 49 bits in LoTTA. Although only visible in mips and motion, the conditional branches used in PeLoTTA typically affect code efficiency as they are not always scheduled in an optimal fashion. The predicated execution in LoTTA typically results in more efficient code.

The effect of IRF on the compiled code size was similar with the basic IRF and the improved IRF. Table 5 lists the code sizes for each benchmark on different sizes of the improved IRF. Code size with IRF is slightly larger than without IRF, due to the additional header instructions required for starting IRF execution. In coremark, gsm and jpeg the code size is smaller with IRF. This likely results from a different scheduling result due to the chaotic nature of tcecc in some cases.

\subsection{Clock Frequency}

Timing results are presented in Table 6. To evaluate the potential for maximizing serial performance, the LoTTA and LatticeMico32 were synthesized with target clock frequencies at intervals of $0.05 \mathrm{~ns}$ in order to find the maximum clock frequency allowed by the ASIC technology. LoTTA reached a maximum clock frequency of $1333 \mathrm{MHz}$ and LatticeMico32 reached $1667 \mathrm{MHz}$. The ALU output port in LoTTA can be used directly for predicated execution, and thus reduce need for branching, but the trade-off becomes visible in the critical timing path which ended up between the ALU output and the instruction fetch unit. LatticeMico32 utilizes a six-stage pipeline without predication support, allowing a shorter critical path and higher clock frequency in this case.

Between LoTTA and PeLoTTA-LM32, replacing guarded execution with conditional branching and splitting the

Table 5 Performance and code size results for PeLoTTA core with the improved IRF. Code size did not vary significantly between IRF entry amounts.

\begin{tabular}{llllllllll}
\hline & coremark & adpcm & aes & blowfish & gsm & jpeg & mips & motion & sha \\
\hline & Cycle count & & & & & & & & \\
8 & 511517 & 82728 & 34783 & 667430 & 12938 & 2397328 & 27153 & 7762 & 557779 \\
16 & 502962 & 82728 & 34783 & 667560 & 12938 & 2389895 & 27153 & 7762 & 557779 \\
32 & 500691 & 82728 & 34756 & 667560 & 12938 & 2477417 & 27153 & 7763 & 557779 \\
64 & 500864 & 82728 & 34891 & 667239 & 12864 & 2508551 & 27153 & 7767 & 557523 \\
128 & 583237 & 82728 & 34906 & 667239 & 12852 & 2583892 & 27153 & 7784 & 555210 \\
256 & 583304 & 82728 & 34600 & 667986 & 12987 & 2578699 & 27153 & 8169 & 553668 \\
512 & 583304 & 82728 & 34600 & 667986 & 12914 & 2581820 & 27153 & 8688 & 553154 \\
& Code size (kB) & & & & & & & 3.0 & \\
\end{tabular}


Table 6 Timing results for the evaluated cores after place and route on a $28 \mathrm{~nm}$ process technology.

\begin{tabular}{lll}
\hline & $\max C_{f}(\mathrm{MHz})$ & Result after \\
\hline LatticeMico32 & 1667 & Synthesis \\
LoTTA & 1333 & Synthesis \\
PeLoTTA-LM32 & 1670 & Place and route \\
PeLoTTA-ZR & 1790 & Place and route \\
SuperLoTTA & 2630 & Place and route \\
zero-riscy & 1230 & Place and route \\
\hline
\end{tabular}

instruction fetch and decode stages allowed an increase in maximum clock frequency of $25 \%$.

Adding the 3-stage execution pipeline, conditional branching and the additional ALU delay allowed an increase of $97 \%$ in the SuperLoTTA core maximum clock frequency in comparison to LoTTA. The maximum clock frequency of $2630 \mathrm{MHz}$ is enabled by the efficient instruction fetch and decode logic of the TTA programming model, the lightweight interconnection network connecting components inside the core, and the simple register file that still supports the multi-issue capabilities. The critical path ended up inside the latch-based register file.

The effect of adding an IRF to PeLoTTA-ZR on maximum operating clock frequency is presented in Table 7 . As reading instructions from the IRF ends up on the timing critical path, increasing the IRF size decreases the maximum clock frequency. With the smallest size of 8 , the maximum clock frequency is reduced by $15 \%$. With size 512 , the reduction is $37 \%$.

In our previous work, LoTTA core was synthesized with two timing constraints: a relaxed constraint to obtain a low-power design point and a tight constraint for a highperformance design point. Since the $28 \mathrm{~nm}$ technology library includes body-biased variations of the standard cells, a $10 \mathrm{~ns}$ timing constraint was chosen in order to mostly utilize the less energy consuming but slower standard cells. With the $10 \mathrm{~ns}$ constraint, the LoTTA core reached a

Table 7 PeLoTTA-ZR place \& route results with IRF. To obtain the maximum clock frequency, instruction memory timing characteristics modeled with $0.40 \mathrm{~ns}$ hold time for instruction read and $0.15 \mathrm{~ns}$ for address setup time.

\begin{tabular}{lll}
\hline IRF entries & $\max C_{f}(\mathrm{MHz})$ & Cell area $\left(\mu \mathrm{m}^{2}\right)$ \\
\hline 8 & 1612 & 10769 \\
16 & 1538 & 12880 \\
32 & 1515 & 16121 \\
64 & 1351 & 22036 \\
128 & 1266 & 33018 \\
256 & 1266 & 55098 \\
512 & 1136 & 99269 \\
\hline
\end{tabular}

maximum clock frequency of $311 \mathrm{MHz}$ after synthesis, with the timing critical path starting from the ALU predicate output, through RF read into the instruction fetch unit and ending in the program counter register.

With the same configuration, LatticeMico32 reached a maximum clock frequency of $351 \mathrm{MHz}$, with the critical path in the multiplier unit. LoTTA including the basic IRF with 256 entries reached a maximum clock frequency of 221 MHz.

\subsection{Execution Performance}

Benchmark execution cycle counts for the evaluated cores are presented in Table 4 . The data memory accesses are scheduled statically in TCE, so the reported cycle counts are instruction cycles. Due to the zero delay slot branching in LoTTA, its cycle counts are the lowest in all benchmarks except jpeg and motion. A large fraction of execution time in jpeg consists of manually written memcpy operations. Due to a chaotic effect in the TCE compiler between compiling for LoTTA and PeLoTTA, the latter resulted in a significant improvement in execution cycles, as the compiler unrolled the manual memcpy loop, unlike with LoTTA.

Compared to LoTTA, disregarding jpeg not to emphasize the effect of compiler memcpy behaviour, LatticeMico32 requires a geometric average of $1.29 \times$ more cycles to execute the benchmark set. This is explained by the 6-stage pipeline as opposed to the 1-stage of LoTTA, as well as the ILP provided by LoTTA. In addition, predicated execution from RF and directly from the ALU output port help in saving clock cycles. In the control-oriented Coremark, the predicated ALU execution of LoTTA improved the cycle count by $10 \%$. The best speedup $(2.9 \times)$ was obtained in aes. Here the TTA compiler could exploit the instruction-level parallelism in the benchmark very efficiently.

Compared to LoTTA, PeLoTTA uses on geometric average $1.08 \times$ more cycles to execute the benchmarks. This is due to the additional instruction pipelining and conditional branching added to increase the operating clock frequency. Zero-riscy uses $1.34 \times$ more clock cycles compared to LoTTA. Although otherwise matched to zeroriscy, PeLoTTA is able to achieve lower cycle counts due to its multi-issue architecture and aggressive loop unrolling. Zero-riscy performs better in Coremark and mips, which are control-oriented benchmarks, and in the data oriented motion. In Coremark, with IRF sizes larger than 64 entries, the cycle count increases by $16 \%$. This is caused by an inner loop, that does not fit into an IRF of 64 entries and its execution always exits on the second iteration. Due to its structure, this loop requires 99 instructions to be prefilled into the IRF before execution can resume. As the IRF block allocation routine does not in its current form take into account the loop iteration counts even if they are known at 


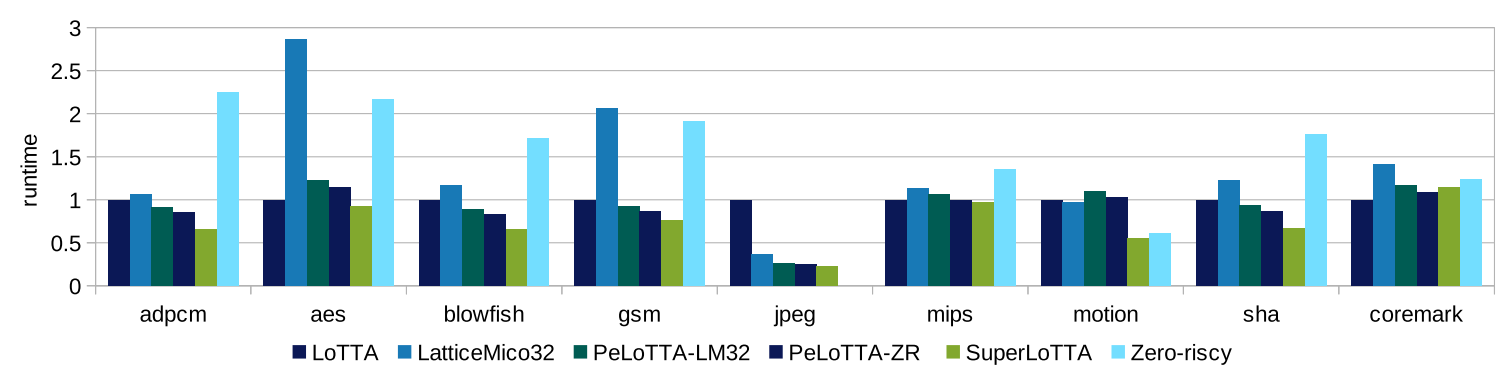

Figure 6 Runtime comparison. Results normalized to LoTTA per benchmark.

compile time, this inner loop always adds 99 stall cycles to execution when it is entered.

SuperLoTTA uses $1.35 \times$ more cycles compared to LoTTA. The largest increase in cycle counts is found in the control-flow-oriented coremark and mips. The increase is explained by the 3 delay slots in branching, conditional branches instead of predicated execution, and the added ALU latency.

On average, SuperLoTTA and zero-riscy consume close to same amount of clock cycles, although SuperLoTTA has a longer branching delay. This is explained by the multi-issue architecture and aggressive loop unrolling in SuperLoTTA. SuperLoTTA used less clock cycles in adpcm, aes, blowfish and sha.

Cycle counts for benchmarks when using PeLoTTA with IRF are listed in Table 5. As the number of IRF entries grows, the cycle count grows. This is explained by the IRF prefilling required for instruction presence assurance, and the stall cycle required when entering and exiting IRF execution. As the number of entries grows, the compiler allocates larger instruction windows into the IRF. This typically leads to increased prefill amount, which increases the cycle count. Also, the current IRF architecture used in TCE always assumes that there is no valid data in the IRF, when starting execution. This increases the cycle count, if prefill is required in an instruction window, that is executed multiple times from the IRF, but the execution switches to instruction memory in between.

Figure 6 presents absolute runtimes for the benchmarks. In nearly all cases, SuperLoTTA with the highest clock frequency is the fastest when measured in absolute time units. Only in Coremark, PeLoTTA with LatticeMico32matched hardware is slightly faster. As seen from Table 4, even though it requires more cycles than any of the other evaluated core, it performs the best when measured in absolute runtime. The increase in cycles and achieved clock frequency stem from the same reason: increased pipelining in the instruction stream and compute units.

Organized in the order of geometric average of the runtimes over the benchmark set (excluding jpeg)), SuperLoTTA is the fastest with $36.7 \mu \mathrm{s}$, followed by PeLoTTA-ZR: $45.0 \mu \mathrm{s}$, LoTTA: $47.7 \mu \mathrm{s}$, PeLoTTA-LM32: $48.7 \mu \mathrm{s}$, LatticeMico32: $66.4 \mu \mathrm{s}$ and zero-riscy: $72.6 \mu \mathrm{s}$.

\subsection{Area}

Area comparison for the evaluated cores after place and route is presented in Fig. 7. LoTTA without IRF is roughly the same size as the LatticeMico32. When incorporating an IRF, the increment in area is quite linear in relation to the number of IRF entries. The entries are implemented as flipflops in the RTL description. In the baseline LoTTA, the RF occupies more than half of the silicon area.

PeLoTTA matched with zero-riscy hardware is the smallest, followed by PeLoTTA matched with LatticeMico32. The difference between the two is mostly due to the latch-based RF being smaller than the flip-flop based, as can be seen from Fig. 7.

SuperLoTTA occupies more area than PeLoTTA due to the added registers for pipelining in the instruction stream,
Figure 7 Cell area $\left(\mu \mathrm{m}^{2}\right)$ distribution after place and route.

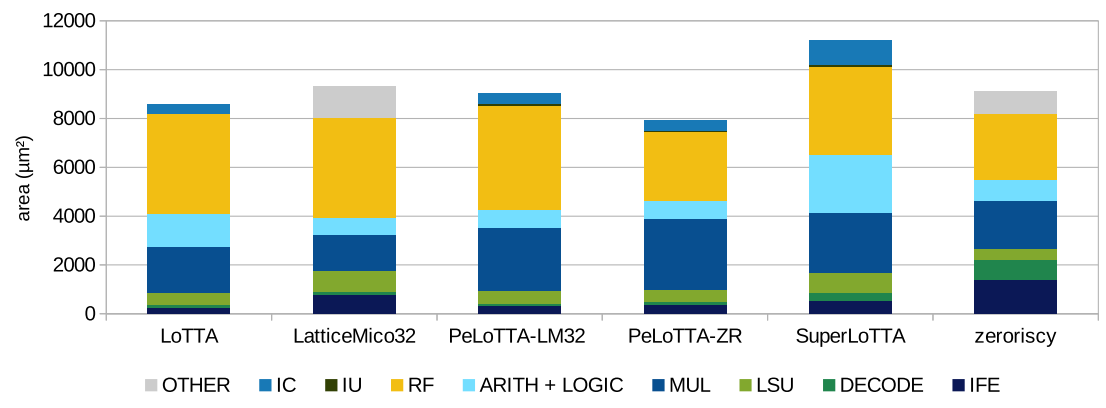


Figure 8 Core energy consumption compared to PeLoTTA-ZR.

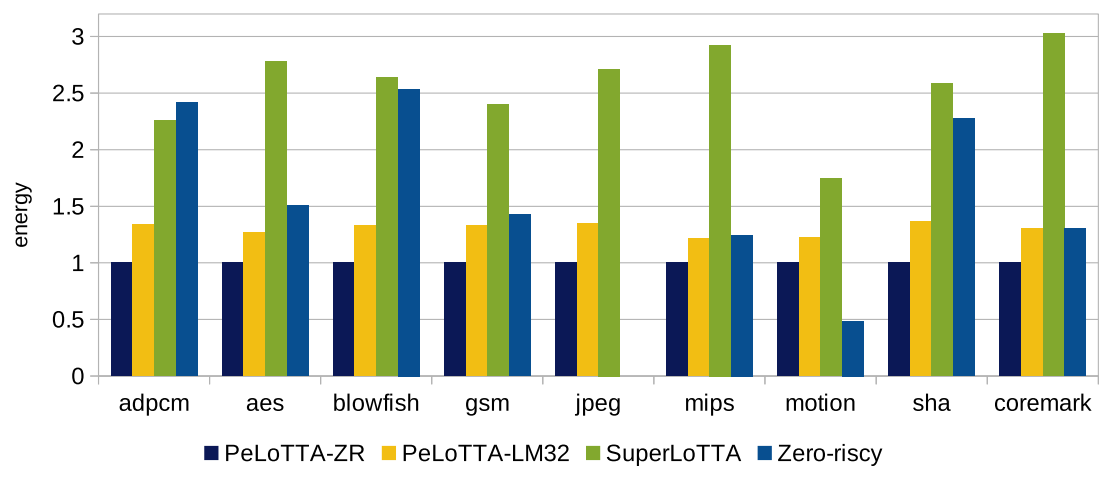

load-store unit and ALU. Also, to achieve the higher clock frequency, the synthesis tool has replaced some cells with more energy-consuming and larger but faster versions.

\subsection{Energy Consumption}

Figure 8 presents the core energy consumption for the benchmark set relative to PeLoTTA-ZR. Here, PeLoTTALM32 with LatticeMico32-matched hardware consumes slightly more energy than PeLoTTA-ZR due to the registerbased RF. Zero-riscy consumes on average 58\% more energy on the benchmarks than PeLoTTA and in the worst case, $175 \%$ more. SuperLoTTA consumes on average $153 \%$ more energy than PeLoTTA and in the worst case 203\% more. In motion, zero-riscy consumes less energy than PeLoTTA-ZR, as it executes the benchmark in $2.5 \times$ less clock cycles.

In order to highlight the execution latency in conjunction with the energy consumption, Fig. 9 presents the energydelay-product (EDP) of the cores relative to PeLoTTA. Here, PeLoTTA achieves $6.8 \times$ better EDP in the best case compared to zero-riscy and $2.5 \times$ on average. This is due to better overall execution cycle counts in the benchmarks, smaller power consumption and higher clock frequency. The difference to LatticeMico32-matched PeLoTTA is negligible, as it has a slightly higher clock frequency, compensating for its higher energy consumption in the EDP results.
PeLoTTA has $2.1 \times$ better EDP in the best case over SuperLoTTA, but on average the difference is not significant.

To evaluate the effect of using different IRF sizes on the instruction stream overall in a processor, we evaluated a system, where the instruction stream hierarchy consists of an on-chip SRAM instruction memory and and IRF. Energy consumption for LoTTA core with different basic IRF sizes is presented in Fig. 10. Here, the combined core and instruction memory energy consumption is presented with an instruction memory size of $16 \mathrm{k}$ entries. As LoTTA has a 49 bit instruction word and 50 bit instruction word with IRF, we equalized the memories between LoTTA and LatticeMico32 not according to the amount of bytes, but number of entries. This takes into account the wider instruction word in LoTTA for a fair comparison. Here, the energy benefit of the IRF increases, as the memory size increases. This is due to the low-power flavour ITRSLSTP SRAMs energy consumption consisting mostly of the number of accesses to them, due to having a low standby power. The instruction SRAM access energy numbers were generated with Cacti-P [28].

Comparing the energy consumption of different IRF sizes with the proportions of instruction executed from either memory or IRF in Fig. 11, it can be seen that with small IRF sizes, when the IRF utilization is low, energy is not saved significantly or not at all. As the amount of IRF entries grows, so does the IRF utilization and the energy
Figure 9 Energy-delay product of cores compared against PeLoTTA-ZR, lower is better.

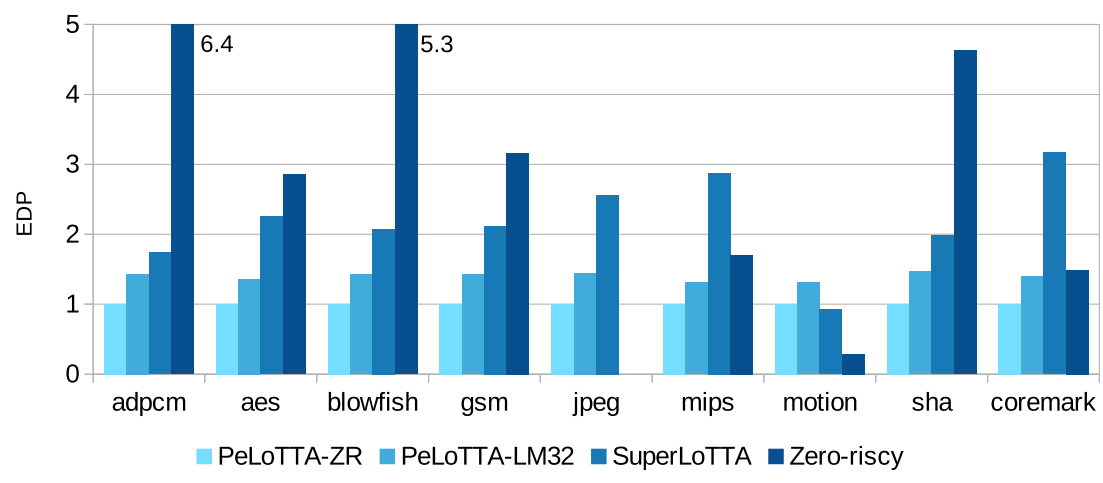




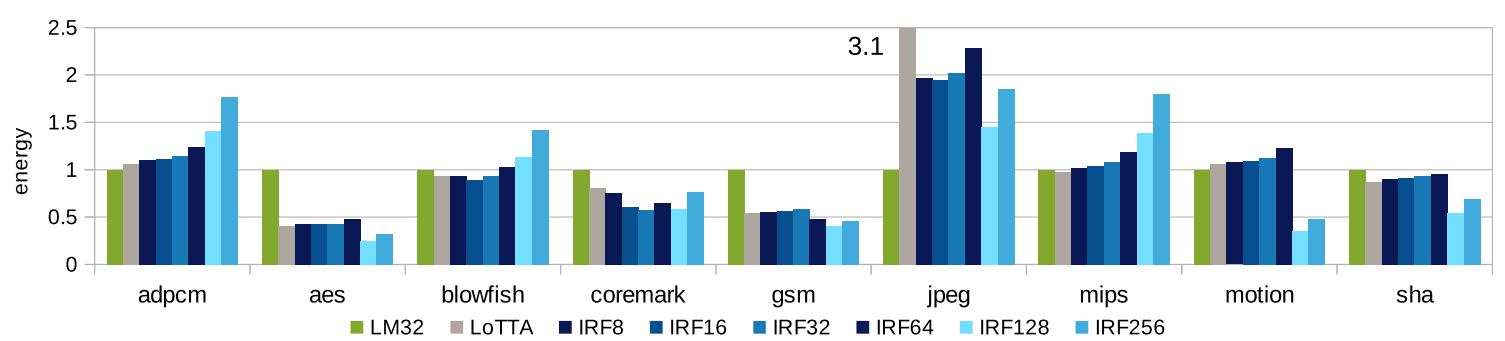

Figure 10 Core + instruction memory energy of LoTTA without and with basic IRF at 200MHz. Each benchmark normalized to LatticeMico32. Instruction memory size is 16k entries (LoTTA: $100 \mathrm{kB}$, LoTTA + IRF: $102 \mathrm{kB}$, LatticeMico32: $64 \mathrm{kB}$ ).

savings. Increasing the number of entries from 128 does not increase the IRF utilization significantly, worsening the energy saving as the larger, but less utilized IRF now consumes more energy.

Figure 12 shows comparison of the combined core and instruction memory energy consumption for zero-riscy, SuperLoTTA and PeLoTTA, which is compared with and without the improved IRF. Compared to SuperLoTTA, in aes, gsm, mips, motion, gsm and coremark, zero-riscy consumes significantly less energy. This is due to the increased cycle count in the benchmarks on SuperLoTTA due to the added pipelining in conjunction with the wider instruction word. In other words, SuperLoTTA reads significantly more bits from the instruction memory, consuming more energy as a trade-off for high clock frequency. At small IRF sizes, as seen from Fig. 13, the benchmarks mostly execute directly from the instruction memory instead of the IRF. Increasing the amount of entries in IRF also increases its utilization. From size 128 onwards, IRF utilization does not increase significantly, except in aes. This behaviour in utilization is translated to energy consumption in Fig. 12. At large IRF sizes, the large amount of entries starts to result in excessive energy consumption, countering the benefits gained from IRF. On geometric average, SuperLoTTA consumes $1.4 \times$ more energy than zero-riscy on the benchmark set.

PeLoTTA without IRF consumes slightly less energy in adpcm and blowfish compared to zero-riscy. In motion, PeLoTTA requires $2.5 \times$ more cycles to execute the benchmarks, resulting in the significantly higher energy consumption. Comparing the geometric average of energy consumption over the benchmark set, PeLoTTA consumes $42 \%$ more energy compared to zero-riscy.

Although the proposed cores consume less energy compared to zero-riscy in most benchmarks, the zero-riscy compiler is able to utilize a high number of compressed instructions in all benchmarks, leading to good energy-efficiency of the instruction stream. It is left as a future work for us to add instruction compression to further reduce energy consumption in addition to the locality based optimization provided by IRF.

Finally, evaluation of energy-delay product in a processor system with an on-chip instruction memory with either zeroriscy, PeLoTTA or SuperLoTTA is presented in Fig. 14. SuperLoTTA achieved the best individual benchmark EDP improvement compared to zero-riscy in adpcm, where it's EDP was $3.0 \times$ lower. On a geometric average over the full benchmark set, SuperLoTTA achieved and EDP within $1 \%$ difference to zero-riscy. In Coremark, mips and motion, SuperLoTTA had higher EDP compared to zero-riscy, due to using more clock cycles and, thus, increasing the instruction memory energy consumption as more instructions were fetched. In these cases, the higher clock frequency of SuperLoTTA could not compensate for the increased energy consumption, leading to higher EDP than zero-riscy.

PeLoTTA achieved a geometric average of $16 \%$ better EDP than zero-riscy. Like SuperLoTTA, it had worse EDP in Coremark, mips and motion. As the IRF size was increased,

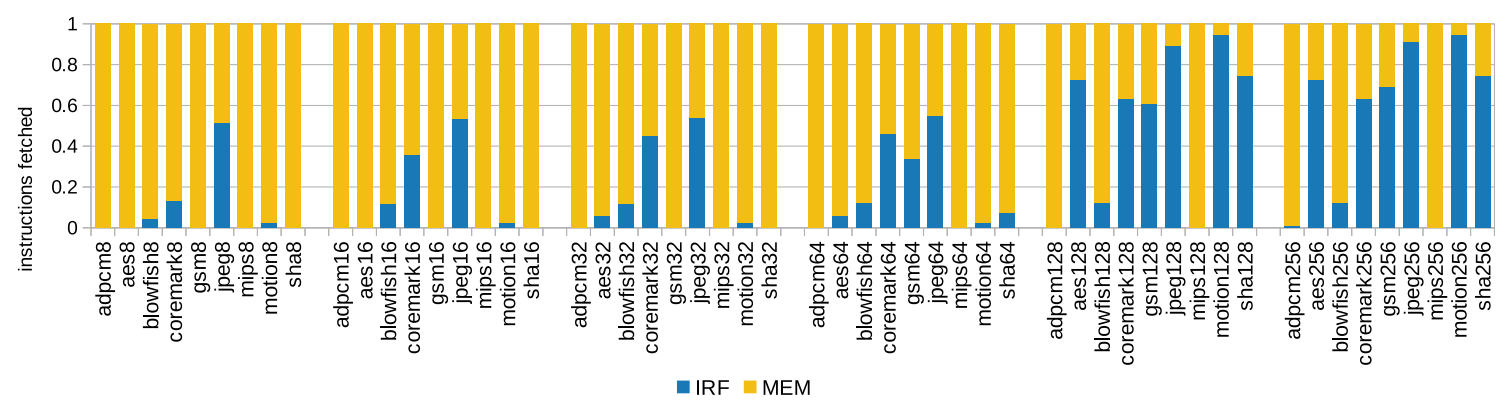

Figure 11 Fraction of instructions fetched from memory and from the basic IRF in LoTTA. 


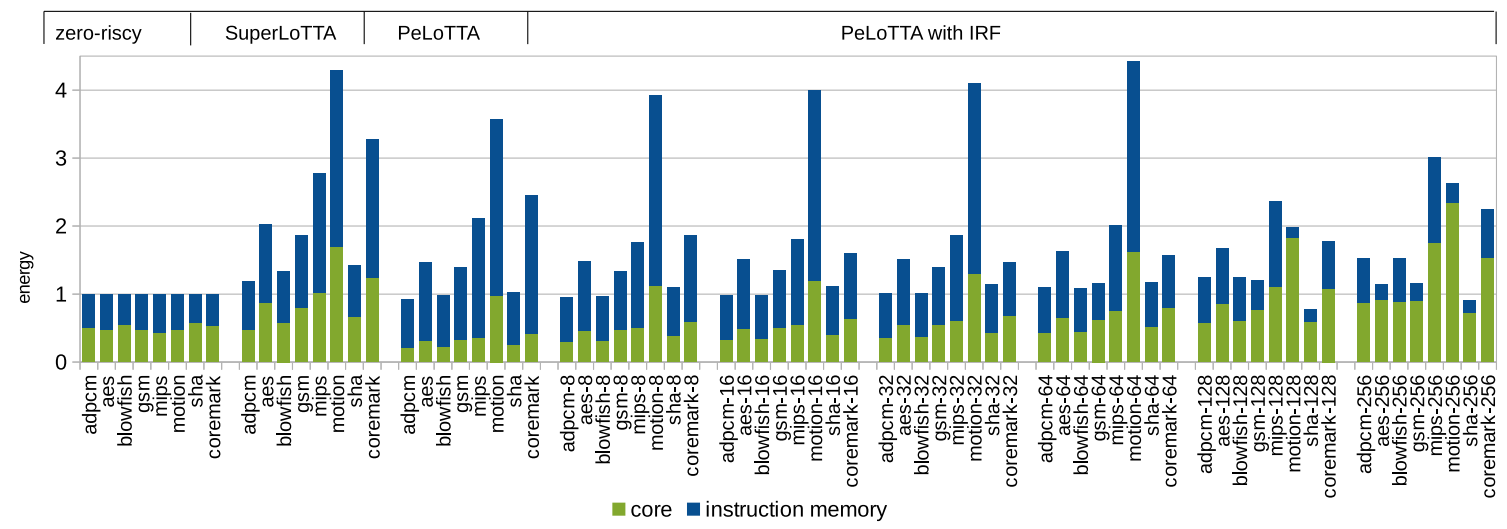

Figure 12 Comparison of processor energy consumption with adpcm, aes, blowfish, coremark, gsm and sha. Numbers normalized to zero-riscy. Instruction memory of 8192 entries, $32 \mathrm{kB}$ on zero-riscy, $44 \mathrm{kB}$ on SuperLoTTA and $46 \mathrm{kB}$ on PeLoTTA.

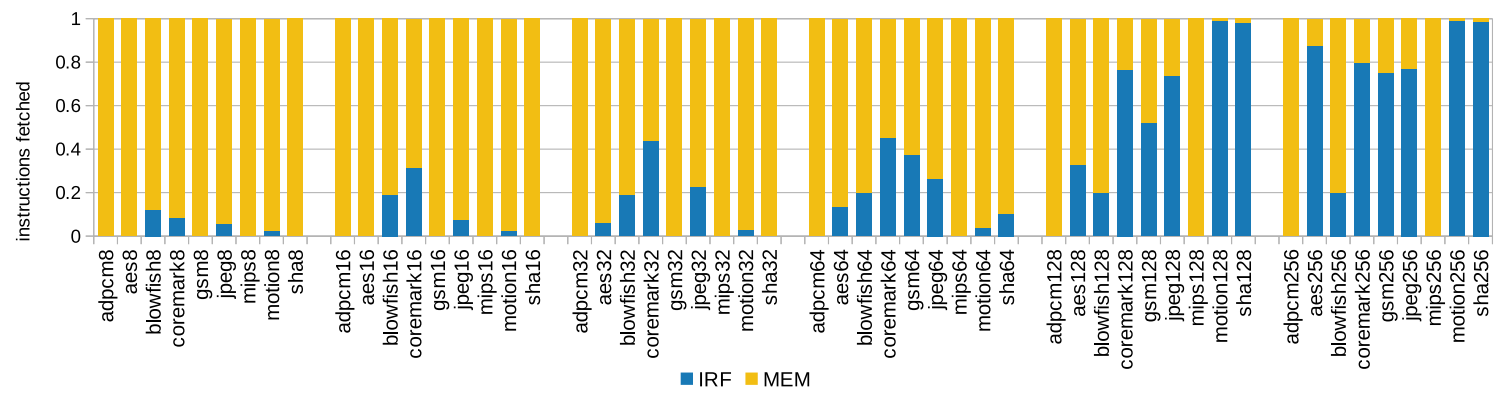

Figure 13 Instructions fetched from memory and from the improved IRF in PeLoTTA. Instruction memory has $16 \mathrm{k}$ entries.

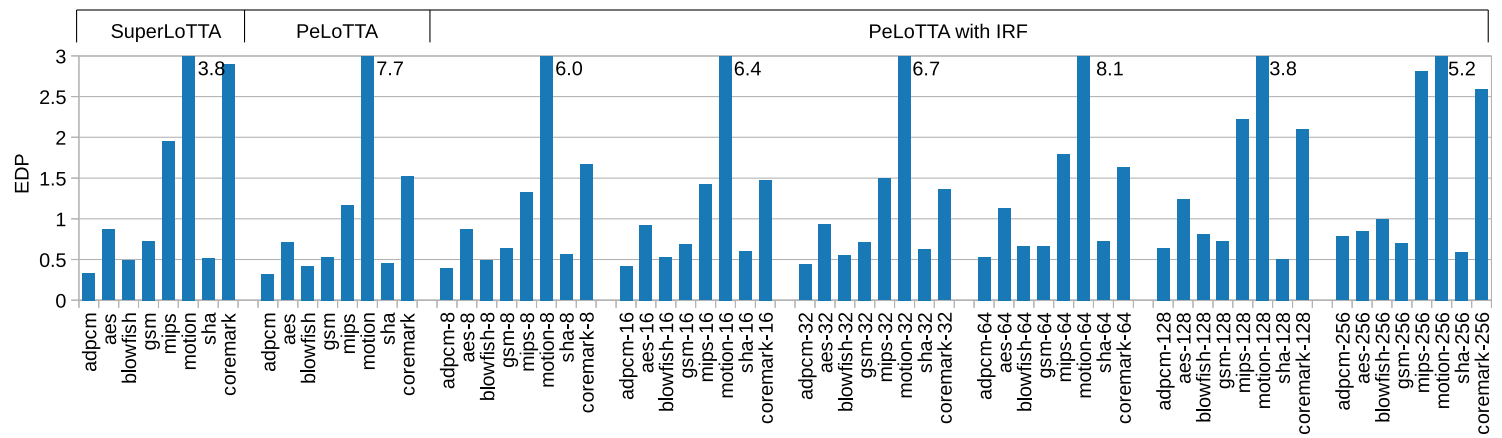

Figure 14 Relative energy-delay product, normalized to zero-riscy with an instruction memory of 8192 entries. Lower is better. 
Table 8 Comparison of the proposed cores with related work.

\begin{tabular}{llllll}
\hline & Technology & Max. $C_{f}(\mathrm{MHz})$ & $V_{d d}(\mathrm{~V})$ & $\mu \mathrm{W} / \mathrm{MHz}$ & $\mathrm{pJ} / \mathrm{cycle}$ \\
\hline SleepWalker [10] & $65 \mathrm{~nm}$ & 25 & $0.32-0.48$ & 7.0 & 2.6 \\
zero-riscy [7] & $65 \mathrm{~nm}$ & 560 & $0.8-1.2$ & $2.3-11.0$ & \\
PeLoTTA-ZR & $28 \mathrm{~nm}$ & 1790 & 0.95 & 14.1 & 4.4 \\
SuperLoTTA & $28 \mathrm{~nm}$ & 2630 & 0.95 & 63.6 & 9.2 \\
\hline
\end{tabular}

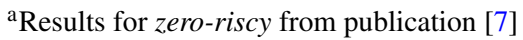

the EDP increased, as the maximum clock frequency decreased as illustrated in Table 7. Simultenously, energy consumption of the IRF increased and while the IRF utilization improved with larger IRF sizes, the combined effect was not enough to improve the EDP.

Finally, a comparison of PeLoTTA-ZR and SuperLoTTA is presented in Table 8. Although SleepWalker and zeroriscy have better $\mu \mathrm{W} / \mathrm{MHz}$ values, our proposed cores provide similar order of magnitude for $\mathrm{pJ} /$ cycle values, with significantly higher maximum operating clock frequencies.

\subsection{Analysis and Effect of IRF}

As the IRF header instructions incur a stall of one clock cycle in program execution while the IRF window size is read in the proposed cores, there is overhead in execution time. Similarly, when entering IRF execution, there is a stall of one clock cycle. However, the increase from these stalls in all benchmarks was less than $0.8 \%$ and had no significant impact on performance.

With PeLoTTA and the improved IRF, cycle counts when using the improved IRF were in many cases slightly lower than PeLoTTA without IRF. This is due to the additional instruction pipeline register in PeLoTTA, that does not exist in LoTTA. If a section of code executes directly from instruction memory, there is always a delay of one clock cycle when branching. Depending on the program, the compiler delay slot filler may not be able to fill in useful instruction to these delay slots. If the same section of code is executed from IRF, branching inside the IRF has no delay. With 8 IRF entries, only blowfish and jpeg used more clock cycles with IRF in the design. From IRF size 128 onwards, also coremark starts using more cycles with than without the IRF.

Due to the code structures in the benchmark programs, utilization of the IRF is low, when IRF size is less than 128, as seen in Figs. 11 and 13. In mips, the IRF is never used, due to a large while loop containing all the code in the benchmark. This loop cannot be split by the TCE compiler to fit into the IRF. As similar structure is found in adpcm, where an encoding and a decoding function are executed within a for loop and do not fit into the IRFs evaluated. An optimization to improve the utilization and reduce energy consumption, would be allowing execution of code sequences such as loops from both the IRF and the next level of memory hierarchy consequently. In the current IRF design, if there is a loop that does not fully fit into the IRF, the compiler does not utilize the IRF at all. Partial execution of loops in this manner from IRF would reduce the energy consumption.

With the smallest basic IRF configuration, eight entries, the IRF is only utilized efficiently in jpeg. This is due to a heavily executed loop in the code containing exactly eight instructions. Compared to the basic IRF, due to replacing predicated branching with conditional branch instruction and different instruction scheduling, the improved IRF is not used as efficiently in jpeg with small IRF sizes. Both IRFs are mostly used in the benchmark set, when it has 128 entries. At this point, in jpeg and motion nearly all code structures fit into the IRF. Doubling the IRF size to 512 entries does not notably increase the IRF utilization.

\subsection{Discussion}

As previous work [23] has extensively studied the energy benefits of the instruction register files compared to small filter caches, and concluded that IRF can save energy over an already effective filter cache, we did not make comparisons to caches in this work.

Although an important design consideration for IoT devices, we left the optimization of standby energy consumption outside the scope of this article, as techniques such as retention registers and power gating are considered generic enough to be adapted on top of our approach as well.

As the IRFs are mapped as registers in the ASIC technology, and in the synthesis flow we used a simple clock gating scheme, where all the registers are gated using the same condition, there is likely room for implementing hierarchical clock gating to the IRF and doing so, decreasing the energy consumption.

As some of the benchmarks have no or poor utilization of IRF, for a processor system it would make sense to implement power gating to the IRF for these cases. These benchmarks should be compiled not to use the IRF, and executed directly from the instruction memory, powering off the costly registers in the IRF. 


\section{Conclusions}

In this paper, we proposed three cores targeting mixed control flow and data processing applications in internetof-things always-on devices. We evaluated design points targeting maximum clock frequency for high throughput tasks and energy-delay product for energy and delay critical tasks. We compared against two RISC cores, LatticeMico32 and zero-riscy.

The TTA programming model alone saved on geometric average $60 \%$ of core energy compared to zero-riscy. As the most obvious drawback of the transport-triggered programming model used in the proposed cores is the larger program size, we designed, optimized and integrated an instruction register file to act as the first-level instruction store. This allowed us to mitigate the effect of the wide instruction word, allowing us to combine the excellent data path energy-efficiency of transport-triggered architectures with an energy efficient instruction supply.

Even though our proposed cores consumed less energy compared to zero-riscy, due to their long instruction word, the energy consumption was higher in a system including an instruction memory. However, in the case of an energyoptimized design point, we achieved on average $14 \%$ better EDP compared to zero-riscy and a best-case of $68 \%$ better EDP in a processor system with an on-chip instruction memory. In a high-performance design point we achieved $2.1 \times$ higher maximum clock frequency with a similar level of energy-delay product.

Evaluation of only the processor cores without the instruction memory hierarchy motivates further research on optimizing the instruction stream, as our energyoptimized core achieves nearly $2.5 \times$ improvement in energy consumption and an $8 \times$ improvement in energy-delay product in the best case. In addition to the IRF, we plan to investigate instruction compression to further mitigate the overhead of the instruction stream. Other plans for future work include moving even more of the control of the IRF from hardware to software, and developing more efficient IRF utilization strategies as these are the low hanging fruits for improvement.

Acknowledgements This work is part of the FitOptiVis project [29] funded by the ECSEL Joint Undertaking under grant number H2020ECSEL-2017-2-783162.

Open Access This article is licensed under a Creative Commons Attribution 4.0 International License, which permits use, sharing, adaptation, distribution and reproduction in any medium or format, as long as you give appropriate credit to the original author(s) and the source, provide a link to the Creative Commons licence, and indicate if changes were made. The images or other third party material in this article are included in the article's Creative Commons licence, unless indicated otherwise in a credit line to the material. If material is not included in the article's Creative Commons licence and your intended use is not permitted by statutory regulation or exceeds the permitted use, you will need to obtain permission directly from the copyright holder. To view a copy of this licence, visit http:// creativecommonshorg/licenses/by/4.0/.

\section{References}

1. Huawei Technologies, Anders, S., Andrae, G. (2017). Total consumer power consumption forecast. Presentation available: https://www.researchgate.net/publication/320225452_Total_ Consumer_Power_Consumption_Forecast.

2. Belkhir, L., \& Elmeligi, A. (2018). Assessing ICT global emissions footprint: trends to 2040 \& recommendations. Journal of Cleaner Production, 177, 03.

3. Magaki, I., Khazraee, M., Gutierrez, L.V., Taylor, M.B. (2016). Asic clouds: specializing the datacenter. In Proceedings of the international symposium on computer architecture (pp. 178-190).

4. Khazraee, M., Zhang, L., Vega, L., Taylor, M.B. (2017). Moonwalk: NRE optimization in asic clouds. SIGARCH Computer Architecture News, 45(1), 511-526.

5. Silven, O., \& Jyrkkä, K. (2007). Observations on power-efficiency trends in mobile communication devices. EURASIP Journal on Embedded Systems, 2007(1), 056976.

6. Lattice Semiconductor. Latticemico32, May 2018. http:// www.latticesemi.com/en/Products/Design/SoftwareAndIP/ IntellectualProperty/IPCore/IPCores02/LatticeMico32.aspx.

7. Schiavone, P.D., Conti, F., Rossi, D., Gautschi, M., Pullini, A., Flamand, E., Benini, L. (2017). Slow and steady wins the race? A comparison of ultra-low-power risc-v cores for internet-of-things applications. In Proceedings of international symposium on power and timing modeling, optimization and simulation (pp. 1-8).

8. Waterman, A., Lee, Y., Patterson, D.A., Asanovic, K. (2011). The risc-v instruction set manual, volume i: base user-level isa. EECS Department, UC Berkeley, Tech. Rep UCB/EECS-2011-62.

9. Multanen, J., Kultala, H., Jääskeläinen, P. (2018). Energy-delay trade-offs in instruction register file design. In Proceedings of the international workshop on signal processing systems, Tallinn.

10. Bol, D., De Vos, J., Hocquet, C., Botman, F., Durvaux, F., Boyd, S., Flandre, D., Legat, J. (2013). SleepWalker: a 25-MHz 0.4V Sub-mm ${ }^{2} 7-\mu \mathrm{m}^{2} \mu \mathrm{W} / \mathrm{MHz}$ microcontroller in $65-\mathrm{nm}$ LP/GP CMOS for low-carbon wireless sensor nodes. Journal of SolidState Circuits, 48(1), 20-32.

11. Klinefelter, A., Roberts, N.E., Shakhsheer, Y., Gonzalez, P., Shrivastava, A., Roy, A., Craig, K., Faisal, M., Boley, J., Oh, S., Zhang, Y., Akella, D., Wentzloff, D.D., Calhoun, B.H. (2015). 21.3 A $6.45 \mu \mathrm{W}$ self-powered IoT SoC with integrated energyharvesting power management and ULP asymmetric radios. In Proceedings of the international solid-state circuits conference (pp. 1-3).

12. Zhang, Y., Xu, L., Yang, K., Dong, Q., Jeloka, S., Blaauw, D., Sylvester, D. (2017). Recryptor: a reconfigurable in-memory cryptographic cortex-M0 processor for IoT. In Proceedings of symposium on VLSI circuits (pp. C264-C265).

13. Senni, S., Torres, L., Sassatelli, G., Gamatie, A. (2016). Non-volatile processor based on MRAM for ultra-low-power iot devices. Journal on Emerging Technologies in Computing Systems, 13(2), 17:1-17:23.

14. Wang, Z., Liu, Y., Sun, Y., Li, Y., Zhang, D., Yang, H. (2015). An energy-efficient heterogeneous dual-core processor for internet of things. In Proceedings of the international symposium on circuits and systems (pp. 2301-2304).

15. Roy, A., Grossmann, P.J., Vitale, S.A., Calhoun, B.H. (2016). A $1.3 \mu \mathrm{w}, 5 \mathrm{pJ} /$ cycle sub-threshold $\mathrm{msp} 430$ processor in $90 \mathrm{~nm} \mathrm{xLP}$ 
FDSOI for energy-efficient IoT applications. In Proceedings of the international symposium on quality electronic design (pp. 158-162).

16. Teittinen, J., Hiienkari, M., Žliobaitè, I., Hollmen, J., Berg, H., Heiskala, J., Viitanen, T., Simonsson, J., Koskinen, L. (2017). A $5.3 \mathrm{pJ} / \mathrm{op}$ approximate TTA VLIW tailored for machine learning. Microelectronics Journal, 61, 106-113.

17. Maxim Corporation (2004). Introduction to the MAXQ architecture.

18. Jääskeläinen, P., Viitanen, T., Takala, J., Berg, H. (2017). HW/SW co-design toolset for customization of exposed datapath processors. In Computing platforms for software-defined radio (pp. 147-164).

19. Hoogerbrugge, J., \& Corporaal, H. (1994). Register file port requirements of transport triggered architectures. In Proceedings of the international symposium on microarchitecture, San Jose (pp. 191-195).

20. Lipovski, G. (1976). The architecture of a simple, effective control processor. In Proceedings of Euromicro symposium on microprocessing and microprogramming (pp. 7-19).

21. Multanen, J., Kultala, H., Jääskeläinen, P., Viitanen, T., Tervo, A., Takala, J. (2018). LoTTA: energy-efficient processor for alwayson applications. In Proceedings of the international workshop on signal processing systems, Cape Town (pp. 193-198).

22. Jääskeläinen, P., Kultala, H., Viitanen, T., Takala, J. (2015). Code density and energy efficiency of exposed datapath architectures. $J$. Signal Process. Syst., 80(1), 49-64.

23. Black-Schaffer, D., Balfour, J., Dally, W., Parikh, V., Park, J. (2008). Hierarchical instruction register organization. Computer Architecture Letters, 7(2), 41-44.
24. Park, J., Balfour, J., Dally, W.J. (2010). Fine-grain dynamic instruction placement for 10 scratch-pad memory. In Proceedings of the international conference on compilers, architectures and synthesis for embedded systems (pp. 137-146).

25. EEMBC-The Embedded Microprocessor Benchmark Consortium (2018). Coremark benchmark May. http://www.eembc.org/ coremark.

26. Hara, Y., Tomiyama, H., Honda, S., Takada, H. (2009). Proposal and quantitative analysis of the CHStone benchmark program suite for practical C-based high-level synthesis. Journal of Information Processing, 17, 242-254.

27. Conti, F., Rossi, D., Pullini, A., Loi, I., Benini, L. (2016). Pulp: a ultra-low power parallel accelerator for energy-efficient and flexible embedded vision. Journal of Signal Processing Systems, 84(3), 339-354.

28. Li, S., Chen, K., Ahn, J.H., Brockman, J.B., Jouppi, N.P. (2011). Cacti-p: architecture-level modeling for sram-based structures with advanced leakage reduction techniques. In Proceedings of the international conference on computer-aided design (pp. 694-701).

29. Al-Ars, Z., Basten, T., de Beer, A., Geilen, M., Goswami, D., Jääskeläinen, P., Kadlec, J., de Alejandro, M.M., Palumbo, F., Peeren, G., et al. (2019). The FitOptiVis ECSEL project: highly efficient distributed embedded image/video processing in cyberphysical systems. In Proceedings of the 16th ACM international conference on computing frontiers. CF' 19 (pp. 333-338).

Publisher's Note Springer Nature remains neutral with regard to jurisdictional claims in published maps and institutional affiliations. 